\section{An adaptive procedure for subjective judgments}

\author{
WALT JESTEADT \\ Human Communication Laboratories \\ Boys Town Institute for \\ Communication Disorders in Children \\ Omaha, Nebraska 68131
}

An adaptive psychophysical procedure is one in which the stimuli presented on a given trial are determined by the observer's responses on previous trials. In recent years, considerable progress has been made in the development of forced-choice adaptive procedures. A variety of these procedures are currently employed in auditory and visual detection and discrimination experiments (Campbell, 1963; Cornsweet, 1962; Kelly \& Lippmann, 1979; Levitt, 1971, 1978; Taylor \& Creelman, 1967; Wetherill \& Levitt, 1965). In auditory psychophysics, the "transformed up-down" procedure described by Levitt (1971) has been the most widely used.

Forced-choice adaptive procedures have several advantages. They automatically select stimuli that are at an appropriate level of difficulty and they provide an estimate of the stimulus difference required for a given level of performance without making strong assumptions about the shape of underlying psychometric functions. One of the most important advantages of adaptive procedures is the way in which they structure the task for the observer. Each block of trials can begin with a few easy trials that demonstrate to the observer the nature of the discrimination task. The difficulty of the test increases automatically as the observer improves. A lapse of attention can cause a rapid increase in the stimulus difference to be discriminated followed by a more gradual return to a more difficult level. Occasional deviations of this kind do not have much influence on the final threshold estimate.

Because decision rules for the selection of stimulus values are typically stated in terms of objective correct or incorrect responses, adaptive procedures generally are not used in experiments that require loudness balances, pitch matches, or other judgments that are inherently subjective, i.e., for which there is no objective criterion for the "correctness" of a response. This is unfortunate, since tasks that involve subjective judgments often have greater problems concerning stimulus selection and observer training than those that involve objective judg-

Thanks are due to W. Kelly, J. Lehman, R. Lippmann, D. Neff, C. Watson, and C. Wier for comments on earlier drafts of this paper. Several lengthy discussions with $C$. Watson were particularly helpful. This work was supported by grants from the NIH Division of Research Resources and NINCDS and by the Boys Town Institute for Communication Disorders in Children. ments. They are difficult for both the experimenter and the observer and could benefit greatly from the advantages associated with forced-choice adaptive procedures. This paper describes one approach to the use of such procedures in subjective-judgment tasks.

\section{Conventional Procedures}

The method of constant stimuli, the method of adjustment, and the method of limits are generally used in situations in which responses cannot be classified as correct or incorrect. The method of constant stimuli (also referred to in the literature as the method of constant stimulus differences or the method of paired comparisons) provides the most complete description of the observer's responses. The most obvious problem with this procedure is that it is often quite inefficient, because extreme values of the comparison stimuli are all classified in the same way and thus contribute little information about the point of subjective equality. A more appropriate set of comparison stimuli often cannot be selected, however, without preliminary tests. Less obvious problems are the occurrence of context effects associated with the range of stimuli used (e.g., Garner, 1954; Helson, 1964; Levison \& Restle, 1968) and related biases caused by the observer's tendency to use responses equally often (e.g., Pollack, 1964; Stevens \& Galanter, 1957). These biases can only be completely avoided if the comparison stimuli are positioned symmetrically around the point of subjective equality, which is, of course, unknown.

The two other standard procedures, the method of adjustment and the method of limits, could be considered as alternative approaches to the problem of stimulus selection in the method of constant stimuli. Both the method of adjustment and the method of limits avoid response biases due to use of a specific set of stimuli as well as the use of stimuli very distant from the point of subjective equality by selecting stimuli, directly or indirectly, on the basis of the subject's previous responses. Because the procedures are not criterion-free and stimuli are presented in a well-ordered series, how. ever, response biases due to sequential effects can be even greater in these methods than the response biases due to stimulus context in the method of constant stimuli. The best example of this is the perseveration of responses of one kind in the method of limits (Woodworth \& Schlosberg, 1954, p. 197).

There have been recent attempts to incorporate the best features of the method of constant stimuli and the method of limits in one procedure by modifying the set of comparison stimuli used in the method of constant stimuli during the initial portion of the test, based on the subject's decisions (Houtgast, 1977; Jesteadt, Green, \& Wier, 1978). It is impossible, however, to obtain rapid estimates of several points on the psychometric function 
simultaneously, and, without this information, it is difficult to decide whether the range of the test stimuli should be expanded or the midpoint shifted, or both. Both a better approach to sampling the psychometric function and a better decision rule for stimulus selection are needed.

In actual practice, a major problem of all of the conventional procedures is that they require the observer to evaluate stimuli that are close to being subjectively equal to the standard. Observers often have difficulty maintaining a consistent criterion when required to make extremely difficult judgments and often conclude that the decisions they are asked to make are essentially arbitrary. A careful explanation of the fact that there is no objective criterion for a correct response rarely leads to more stable performance. Their task is similar in many ways to that of observers operating near chance in a forced-choice detection procedure. It is not surprising that many of them find subjective-judgment tasks very tedious.

\section{An Adaptive Procedure for Subjective Judgments}

Any decision rule that causes trials to be concentrated at specific points on a psychometric function (e.g., Levitt, 1971; Smith, 1961) can be used in tasks involving subjective judgments to select comparison stimuli efficiently, while avoiding response biases. Although Levitt (1971, p. 474) described this approach briefly, it has not been widely used. We have found a forced-choice adaptive procedure to be a considerable improvement over the classical techniques described in the previous section, particularly when the decision rule does not continually select stimuli near the point of subjective equality but focuses instead on points above and below it. To do this, we use two randomly interleaved independent sequences of trials with different decision rules. One sequence of trials begins with variable stimuli that are clearly above the point of subjective equality and the other sequence with variable stimuli that are clearly below it. As in the traditional methods, the observer chooses between two subjective labels in responding to each successive stimulus. The selection of stimuli by the adaptive procedure is governed only by the observer's use of the two subjective response categories, not by how the responses relate to the objective properties of the stimuli.

Consider an example in which we wish to determine the intensity of a $250-\mathrm{Hz}$ tone required for the loudness of that tone to match the loudness of a $1,000 \mathrm{~Hz}$ tone presented at $40 \mathrm{~dB}$ SPL. We might begin by presenting the $250-\mathrm{Hz}$ tone at $30 \mathrm{~dB}$ SPL (sequence A) or $60 \mathrm{~dB}$ SPL (sequence B). The observer would be given the $1,000-\mathrm{Hz}$ standard followed by one of these two alternatives and asked to indicate whether the comparison tone sounded louder or softer than the standard. Following the same version of the Levitt rule that is commonly used in objective detection tasks, two consecutive responses of softer to the stimulus from Sequence A would cause the intensity of the stimulus to be increased on subsequent sequence-A trials. A single response of louder would cause the stimulus intensity to be decreased. Application of this rule across all of the sequence-A trials causes the intensity of the $250-\mathrm{Hz}$ tone presented on these trials to be adjusted to a level at which it is judged softer than the standard $71 \%$ of the time and louder than the standard $29 \%$ of the time. The same rule is used for the randomly interleaved sequence-B trials, except that in this case, the intensity of the $250-\mathrm{Hz}$ tone is decreased following two consecutive responses of louder and increased following a single response of softer. The intensity of the $250-\mathrm{Hz}$ tone presented on these trials is therefore adjusted by the procedure to a level at which it is judged louder than the standard $71 \%$ of the time.

The procedure can be terminated either after a fixed number of trials in each sequence or after a specified number of reversals in the direction of the changes in level within each sequence. The final estimates of the two $71 \%$ points (the levels judged louder and softer than the standard $71 \%$ of the time) are obtained, as in other applications of the Levitt procedure, by averaging the reversal points within each sequence. The point of subjective equality is then estimated by simply averaging the stimulus values at the $71 \%$ points.

Loudness judgments have been used as an example because they are probably the most commonly obtained subjective judgments in psychoacoustic research. We have used the procedure more extensively, however, to obtain lateralization judgments in experiments designed to measure the effective level of suprathreshold tones partially masked by noise (Jesteadt \& Javel, 1978). A trial consisted of a single presentation interval in which a $350-\mathrm{msec}$ noise was presented to one ear and a $150-\mathrm{msec}$ tone was presented to both ears, with the tone centered temporally in the noise. The intensity of the tone in the contralateral ear began either $20 \mathrm{~dB}$ above or below the fixed intensity of the tone in the noise. Following the presentation interval, the observer indicated whether the tone was lateralized to the right or left of the center of the head. The procedure was used to obtain estimates of the contralateral-tone levels that would result in the tonal image being lateralized to the left $71 \%$ of the time and to the right $71 \%$ of the time. These two levels were then averaged to estimate the level required for a centered image. Control conditions were run without noise to test for possible biases in lateralization judgments. Examples of the two sequences of intensities selected by the procedure in a 50-trial block for a control condition and a 100-trial block for a noise condition are shown in panels $A$ and $B$ of Figure 1. Note that in both panels, the two sequences begin at 90 and $50 \mathrm{~dB}$ SPL, equidistant from the fixed-intensity tone, but that one sequence in panel B shifts rapidly to a much lower intensity, reflecting the reduction of the effective level of that tone by the noise. The estimated point of subjective equality is $69.6 \mathrm{~dB}$ in the control condition and 


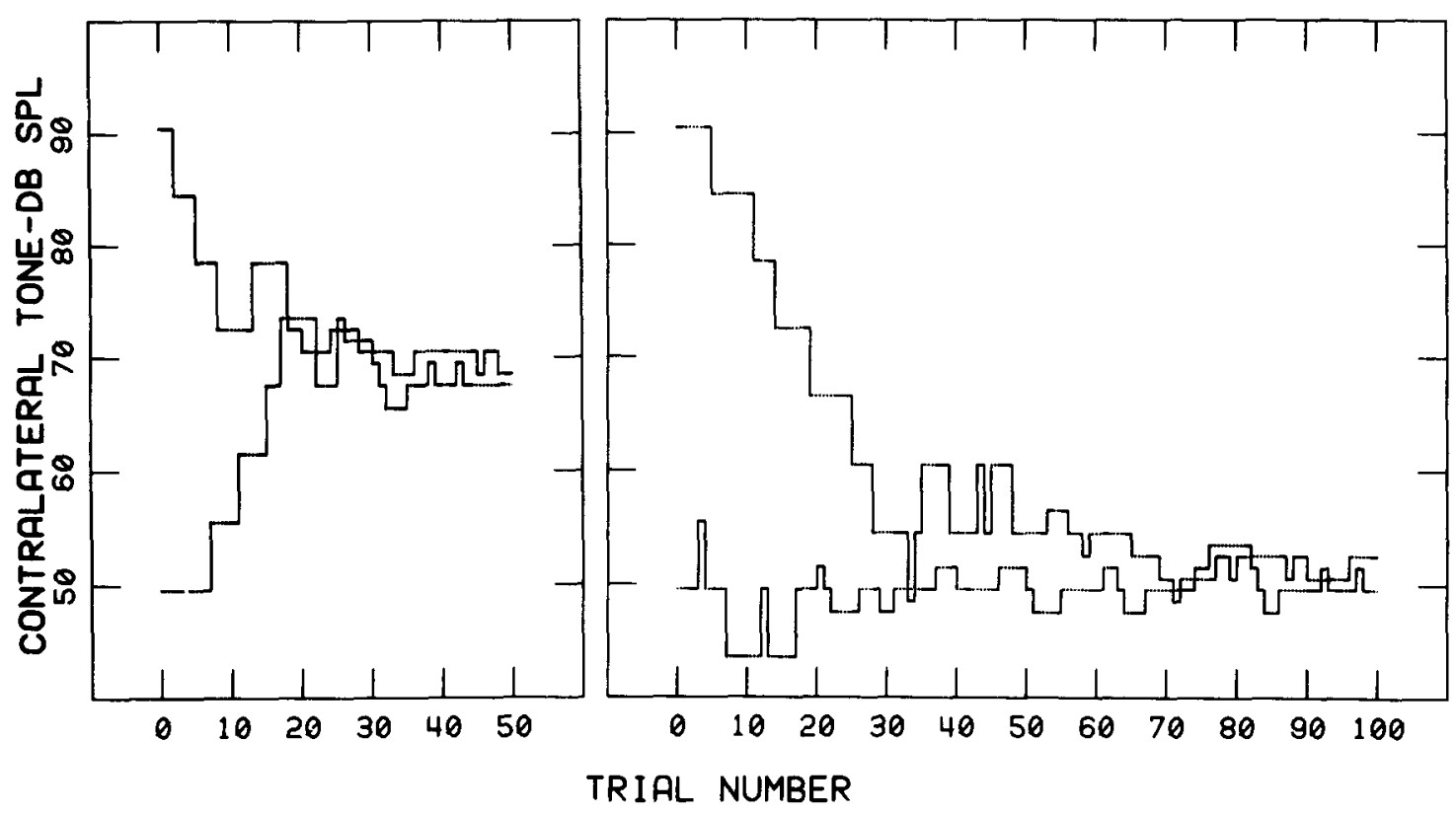

Figure 1. Randomly interleaved sequences of trials in the lateralization procedure. The upper sequences have been shifted up $.5 \mathrm{~dB}$ and the lower sequence down $.5 \mathrm{~dB}$ to separate them at points where they cross. Trials occurring in a sequence are represented by solid lines. Dashed lines appear at these points in the opposite sequence. The sequences in panel $A$ are for a control condition in which a fixed-intensity $2,000-\mathrm{Hz}$ tone was presented to one ear in quiet at $70 \mathrm{~dB}$ SPL. The sequences in panel B were obtained under identical conditions except that a $45-\mathrm{dB}$ spectrum level noise, high-pass filtered at $2,500 \mathrm{~Hz}$, was added to the fixed-intensity tone.

$51.2 \mathrm{~dB}$ in the partial-masking condition. The $29 \%$ and $71 \%$ points are separated by about $3.5 \mathrm{~dB}$ in both cases.

Similar lateralization data have been obtained in recent studies of partial masking or suppression (Bezemer, 1978; Houtgast, 1977; Jesteadt \& Javel, 1978; Kearney, 1979; Sachs \& Zurek, 1979; Zurek \& Sachs, 1979b) and of combination tones (Zurek \& Sachs, 1979a). The majority of these studies have used the method of adjustment to establish the level of the contralateral tone required to produce a centered image, and we began with that procedure. In informal comparisons, we found the adaptive procedure described above to be two to three times faster and considerably more reliable than the method of adjustment.

\section{Discussion}

Several features of this procedure should be noted. First, as shown in Figure 1, the starting points of the two randomly interleaved sequences have little influence on the final outcome. The effect of the starting points has been reduced further by following Levitt's (1971) suggestion that each block of trials begin with a large step size for each sequence, which is then reduced after some number of reversals of the intensity changes within that sequence. Any of the more complex step-size rules discussed by Levitt could, of course, be used. If step sizes and decision rules are selected appropriately, the procedure can be used to track shifts in subjective judgments over time. There is no requirement that the sequence starting points bracket the stimulus value associated with the 50\% point. The use of different starting points on opposite sides of the final $50 \%$ point makes the task clearer to the observer, however, by providing examples of stimuli in each response category at the beginning of each block of trials.

Second, the use of two randomly interleaved sequences greatly reduces the effects of certain response biases. Levitt (1971) recommends the use of interleaved sequences of trials to remove the direct correlation between the accuracy of the observer's response and the difficulty of the task on the next trial. This interleaving is very beneficial in the present procedure because it eliminates the certainty that the stimulus on the next trial will be similar to the current one or that any given response will have a direct influence on the next stimulus. These are the principal sources of the sequential response biases in the method of limits. The response biases due to equal use of response categories in the method of constant stimuli are avoided by the adaptive stimulus selection and symmetric decision rules.

Third, the observer's task is not to match the standard, but to classify stimuli with respect to the standard. The decision rule operates to keep the level of difficulty at a point where $71 \%$ of the judgments are "correct," in the sense that they are consistent with previous judgments. By using a decision rule that maintains two distinctly separate sequences of trials, a task is created in which the observer is, in effect, asked to discriminate between stimuli belonging in one sequence and those belonging in another, using the standard as a reference point. To most 
observers, this appears to be an objective task. We consider that to be one of its greatest advantages.

The primary drawback of estimating the $71 \%$ and $29 \%$ points is that an assumption about the form of the psychometric function is required to obtain an estimate of the $50 \%$ point. In all of our applications to date, we have simply averaged the stimulus values associated with the $29 \%$ and $71 \%$ points. The underlying psychometric functions appear to be linear, and, in any case, the differences between $50 \%$ points estimated by linear interpolation and by the assumption of nonlinear psychometric functions are smaller than the error of measurement. A second drawback in the use of information about the $29 \%$ and $71 \%$ points to estimate the $50 \%$ point is that it is not as efficient statistically as concentrating the trials at the $50 \%$ point (see Levitt, 1978). The loss of efficiency is not great, however, and is far outweighed by the value of presenting the observer with two different types of trials.

In our experience, the procedure is particularly useful in single-interval tasks in which the stimulus is to be compared with a remembered standard. The use of two sequences of trials that ultimately bracket the standard appears to aid the observer in maintaining a consistent representation of the standard itself. Stimuli from one sequence provide a reference point for judging stimuli from the other, and the observer is reminded continually of the distinction to be made between them. The procedure might be very useful, therefore, as a means of obtaining rapid adaptive estimates of category boundaries in studies of categorical perception.

\section{REFERENCES}

Bezemer, A. W. Lateralization applied to masking experiments. I.P.O. Annual Progress Report, 1978, 13, 13-19.

Campbell, R. A. Detection of a noise signal of varying duration. Journal of the Acoustical Society of America, 1963, 35, 1732-1737.

Connsweet, T. N. The staircase method in psychophysics. American Journal of Psychology, 1962, 75, 485-491.

Garner, W. R. Context effects and the validity of loudness scales. Journal of Experimental Psychology, 1954, 48, 218-224.

Helson, H. Adaptation level theory. New York: Harper \& Row, 1964.
Houtgast, T. Phase effects in two-tone suppression investigated with a binaural lateralization paradigm. In E. G. Evans \& J. P. Wilson (Eds.), Psychophysics and physiology of hearing. London: Academic Press, 1977.

Jesteadt, W., Green, D. M., \& Wier, C. C. The RawdonSmith illusion. Perception \& Psychophysics, 1978, 23, 244-250.

Jesteadt, W., \& Javel, E. Measurement of suppression in a simultaneous masking paradigm. Journal of the Acoustical Society of America, 1978, 63, S44(A).

Ke ARnEY, J. K. The temporal course of suppression as measured in a lateralization procedure. Journal of the Acoustical Society of America, 1979, 65, S56(A).

Kelly, W. J., \& Lippmann, R. P. Group-vote rules for adaptive psychophysical testing. Journal of the Acoustical Society of America, 1979, 66, 906-908.

Levison, M., \& Restle, F. Invalid results from the method of constant stimuli.Perception \& Psychophysics, 1968, 4, 121-122.

LevitT, $\mathbf{H}$. Transformed up-down methods in psychoacoustics. Journal of the Acoustical Society of America, 1971, 49, 467-477.

LEvitT, H. Adaptive testing in audiology. In C. Ludvigsen \& J. Barfod (Eds.), "Sensorineural hearing impairment and hearing aids," Scandinavian Audiology Supplement, 1978, 6, 241-291.

Pollack, I. Neutralization of stimulus bias in auditory rating scales. Journal of the Acoustical Society of America, 1964, 36, 1272-1276.

SAChs, R. M., \& ZURE K, P. M. Contralateral-probe measurements of auditory vowel spectra. Journal of the Acoustical Society of America, 1979, 65, S55(A).

Smith, J. E. K. Stimulus programming in psychophysics. Psychometrika, 1961, 26, 27-33.

Stevens, S. S., \& Galanter, E. H. Ratio scales and category scales for a dozen perceptual continua. Journal of Experimental Psychology, 1957, 54, 377-411.

Taylor, M. M., \& Creelman, C. D. PEST: Efficient estimates on probability functions. Journal of the Acoustical Society of America, 1967, 41, 782-787.

Wetherill, G. B., \& LevitT, H. Sequential estimation of points on a psychometric function. British Journal of Mathematical and Statistical Psychology, 1965, 18, 1-10.

Woodworth, R. S., \& SchlosberG, H. Experimental psychology. New York: Holt, 1954.

ZuRek, P. M., \& SaChs, R. M. Combination tones at frequencies greater than the primary tones. Science, 1979, 205, 600-602. (a)

ZUREK, P. M., \& SACHS, R. M. Contralateral-probe measurements of the amplitude suppression and phase shift of one tone caused by another. Journal of the Acoustical Society of America, 1979,65, S55(A). (b)

(Received for publication December 14, 1979; revision accepted April 11, 1980.) 